\title{
A retrospective analysis of renal allograft function and biopsy-proven rejection episodes according to donor type
}

\author{
Paul Martin, Hannah Burton, Paramit Chowdhury, Elham Asgari \\ Guy's and St Thomas' NHS Foundation Trust, London, UK
}

\section{Background}

Renal transplantation is the optimum treatment for the large majority of patients with end stage renal disease. It is well recognised that donor type can have a significant impact on renal allograft outcomes ${ }^{1}$.

The aim of this study was to evaluate the impact of donor characteristics on renal allograft function and rates of rejection in a cohort of recipients from a single, large renal transplantation centre.

\section{Methods}

458 renal allograft recipients who underwent renal transplantation between January 2011 and December 2015 were categorised according to donor characteristics - donation after brain death (DBD), donation after cardiac death (DCD), and live donor (LD).

Delayed graft function (DGF) was defined as the need for haemodialysis within one week of transplantation.

Rejection episodes were categorized according to the Banff classification. Serum creatinine and tacrolimus levels were measured periodically throughout the study period.

\section{Results}

Of the 458 recipients in the study, $132(29 \%)$ received a DBD organ, $111(24 \%)$ a DCD organ, and 215 (47\%) a LD organ.

The rates of DGF according to donor type, and the relationship between DGF and rejection are shown in figure 1. Although LD grafts functioned significantly better than deceased donor (DD) grafts at one year (mean creatinine $126.6 \mathrm{vs} 165.6 \mu \mathrm{mol} / \mathrm{L}$, $\mathrm{p}<0.0001$ ), there was no significant difference between DBD and DCD graft function (mean creatinine $162 \mathrm{Vs} 169.7 \mu \mathrm{mol} / \mathrm{L}, \mathrm{p}=0.48$ ) (Fig 2)

Fig 1. DGF and rejection rates according to donor type

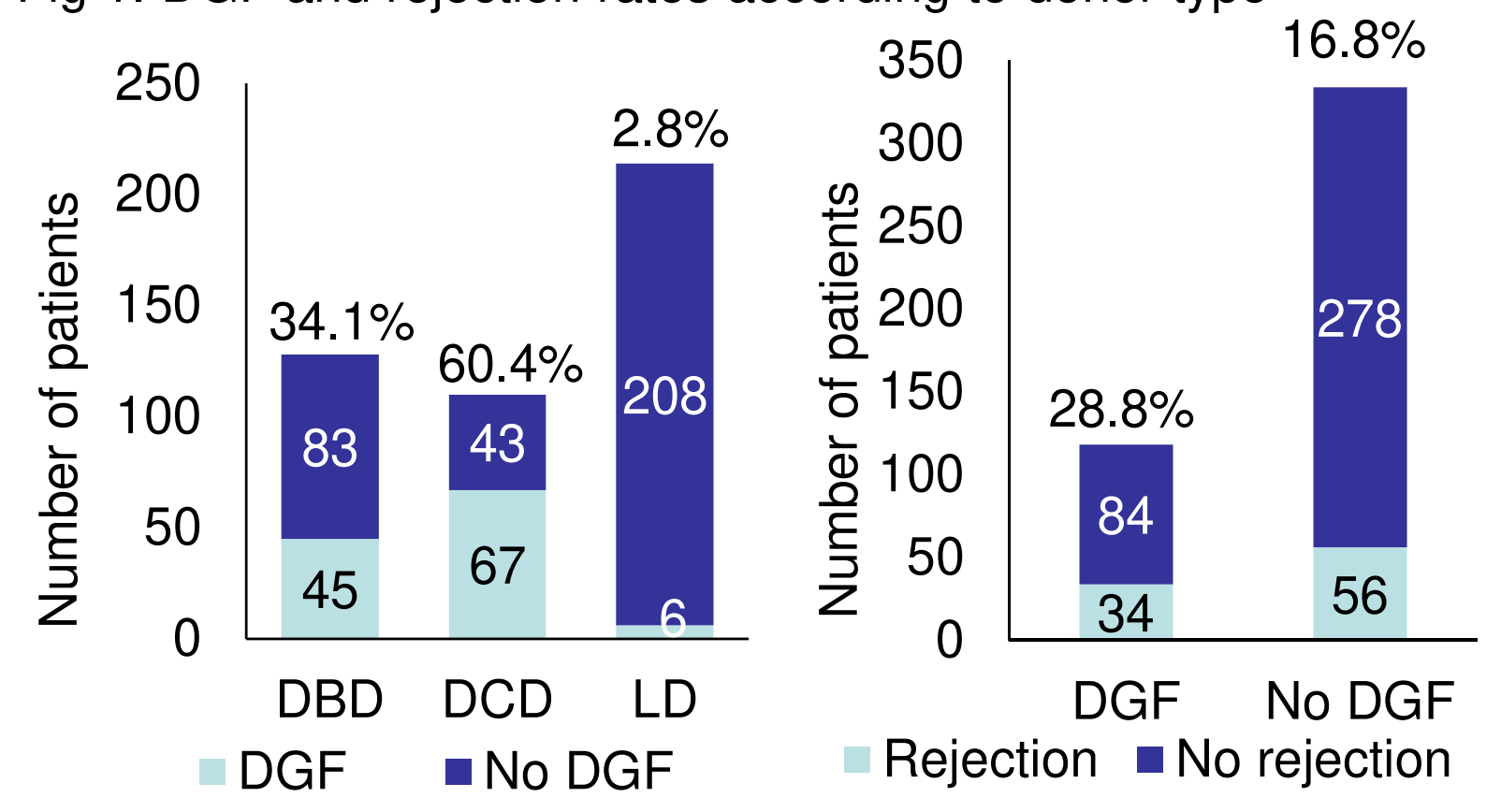

Fig 2. How donor type affects graft function

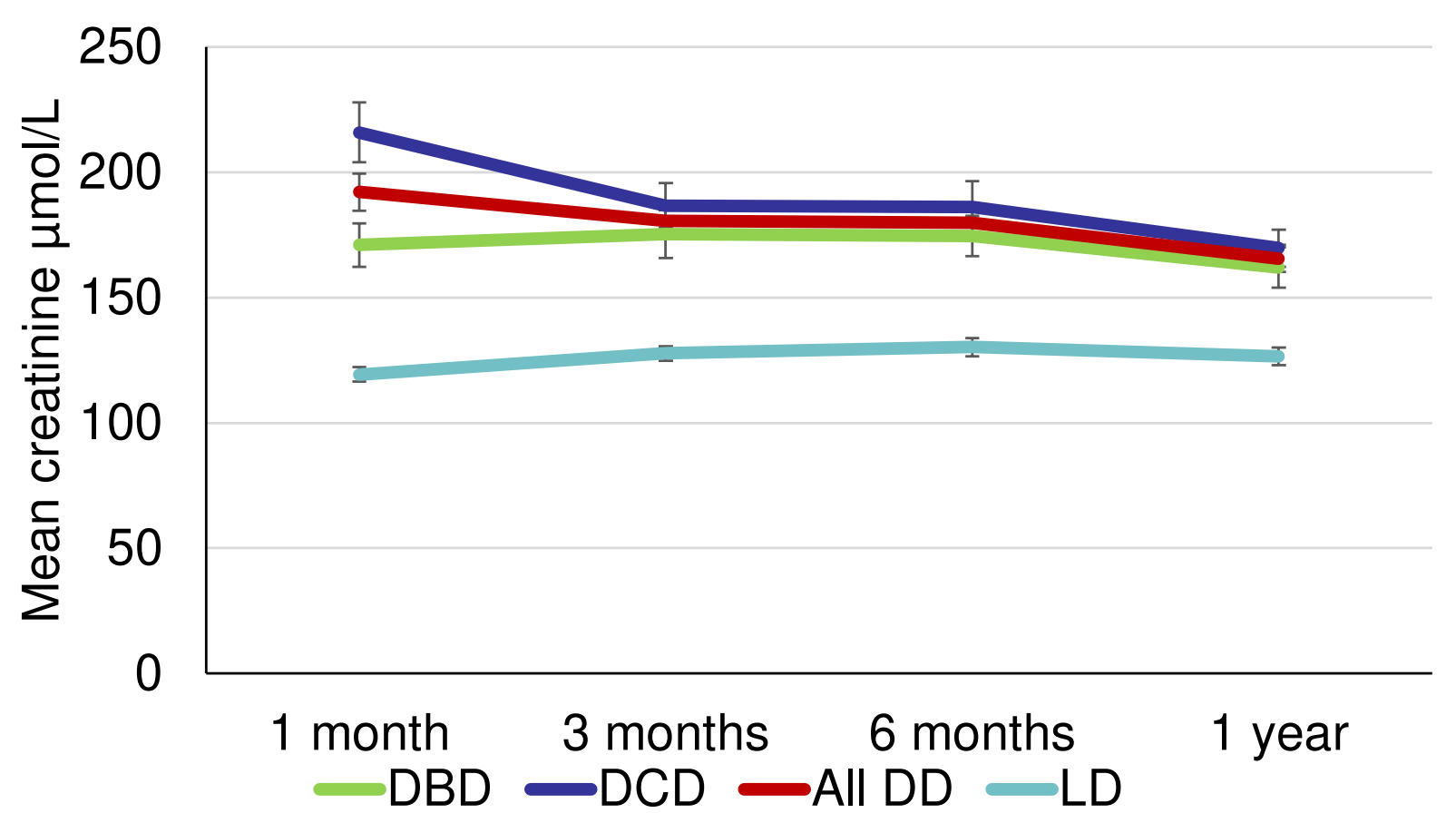

Figure 3 shows the incidence of rejection based on donor type. DD organ recipients had a nonsignificant higher incidence of rejection compared with LD organ recipients $(21.8 \%$ Vs $17.7 \%, p=0.29$ ).

In the DBD group with rejection, mean creatinine at one year was $279.8 \mu \mathrm{mol} / \mathrm{L}$, compared with $185.4 \mu \mathrm{mol} / \mathrm{L}$ in the rejection-free DBD group, whereas in the DCD population the figures were $229.5 \mu \mathrm{mol} / \mathrm{L}$ and $165.5 \mu \mathrm{mol} / \mathrm{L}$ respectively (Fig 4).
Fig 3. How donor type affects rejection

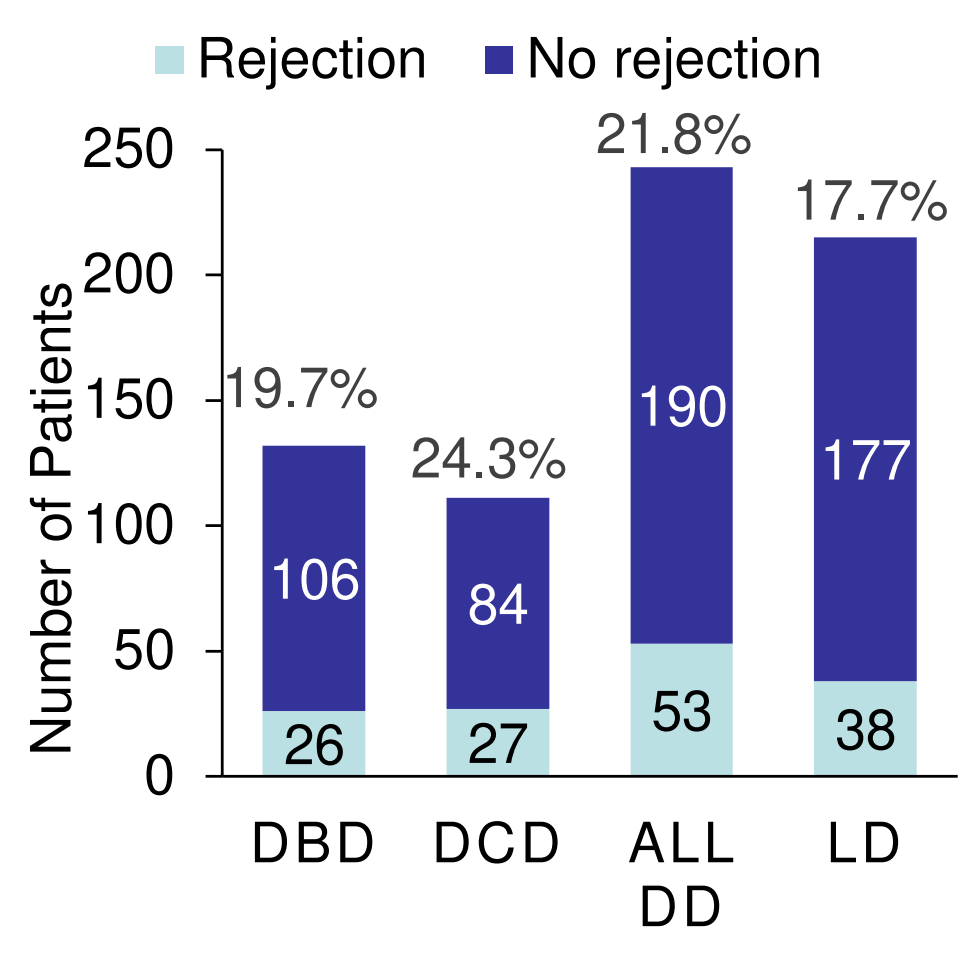

Mean creatinine at one year within the overall DD group with rejection was $252.1 \mu \mathrm{mol} / \mathrm{L}$, compared with $176.6 \mu \mathrm{mol} / \mathrm{L}$ in the rejection-free DD group. Mean creatinine at one year within the LD group with rejection was 187.5 $\mu \mathrm{mol} / \mathrm{L}$ compared with $135.4 \mu \mathrm{mol} / \mathrm{L}$ in the rejection free LD group (Fig 4).

Fig 4. How donor type affects graft function DBD

\section{DCD}

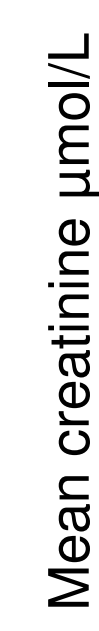

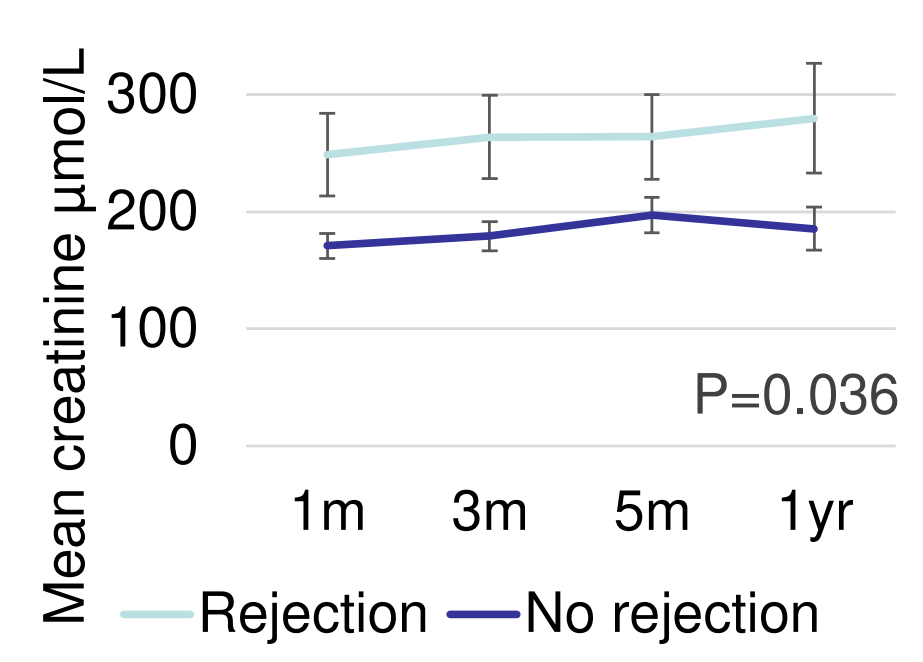

All DD

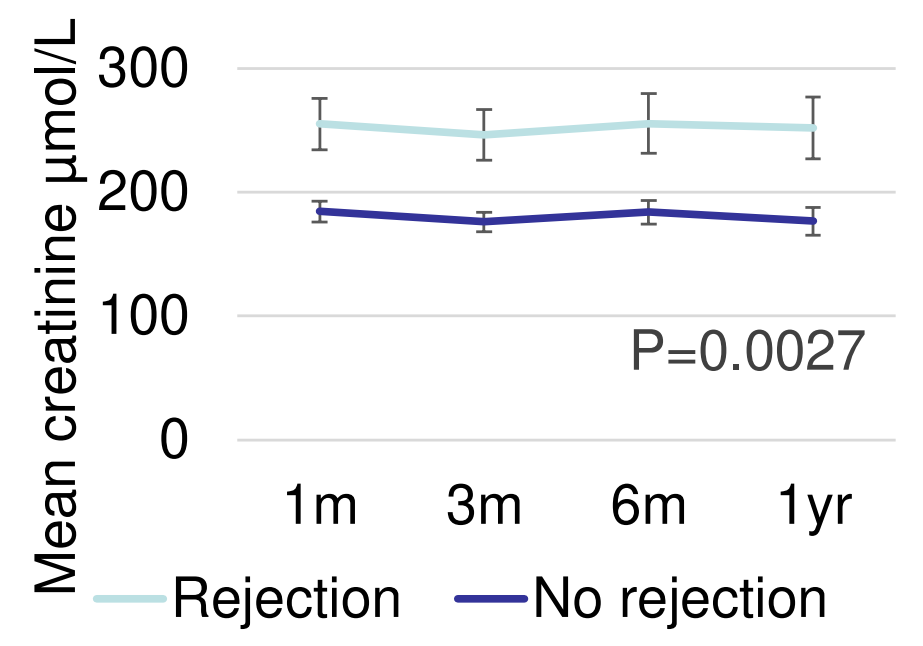

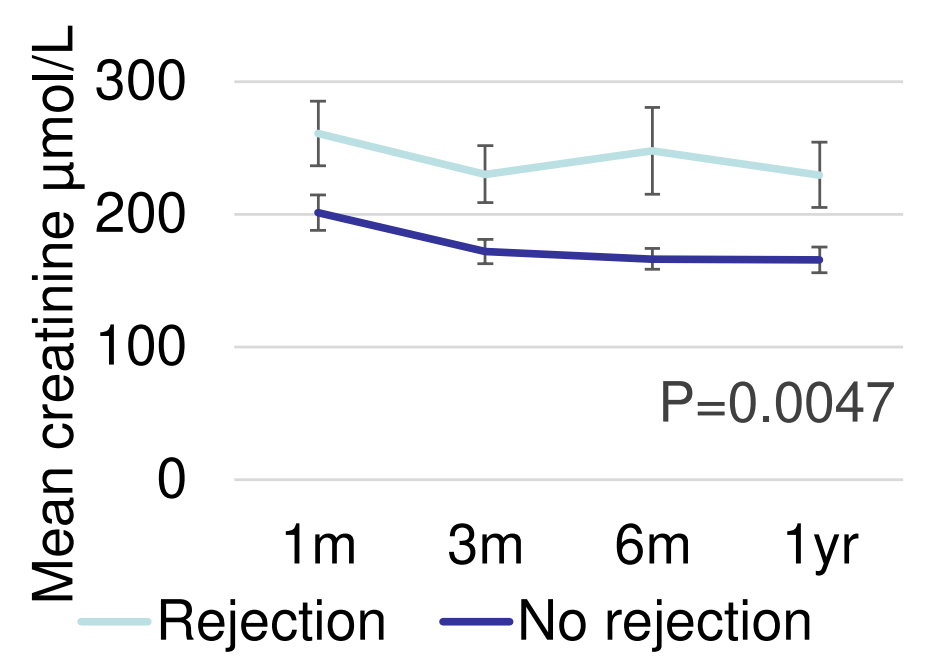

LD

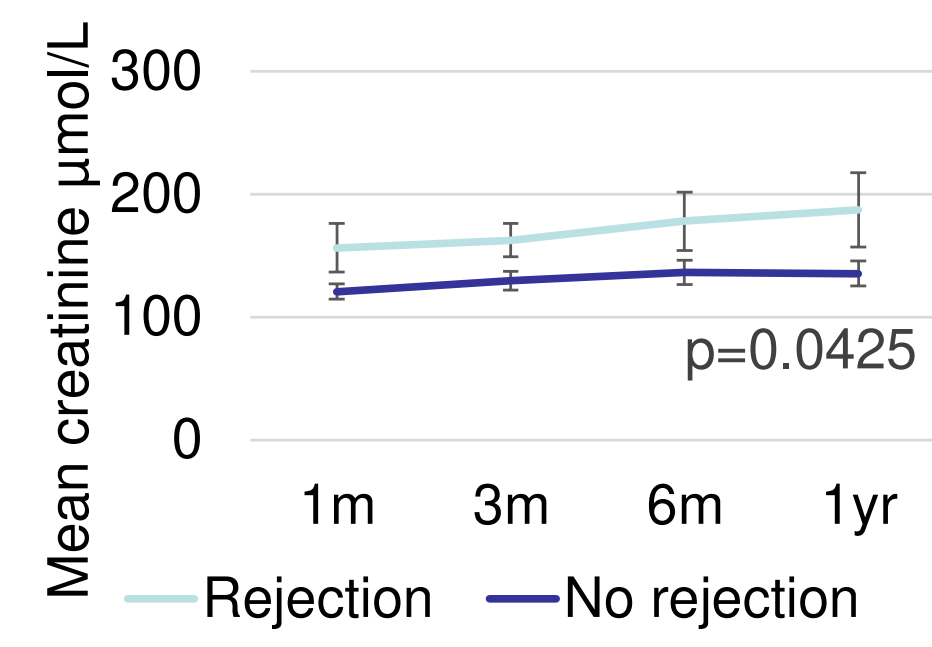

\section{Conclusions}

This single centre study of 458 renal allograft recipients demonstrates that LD allografts function better at one year follow up when compared with all DD allografts.

DGF was more common in the DD allografts, particularly in the DCD group. DGF was associated with an increased risk of rejection. Despite this, DBD and DCD graft functions were comparable at 1 year.

A single episode of rejection resulted in significant allograft impairment at one year follow up across all donor type groups.

\section{References}

${ }^{1}$ Factors influencing long-term outcome after kidney transplantation. C Legendre et al. Transplant International, 2014 Jan; 27(1):19-27

\section{Guy's and St Thomas' N/HS NHS Foundation Trust}

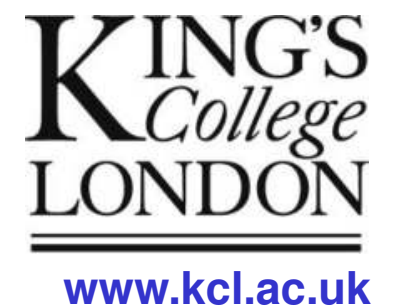

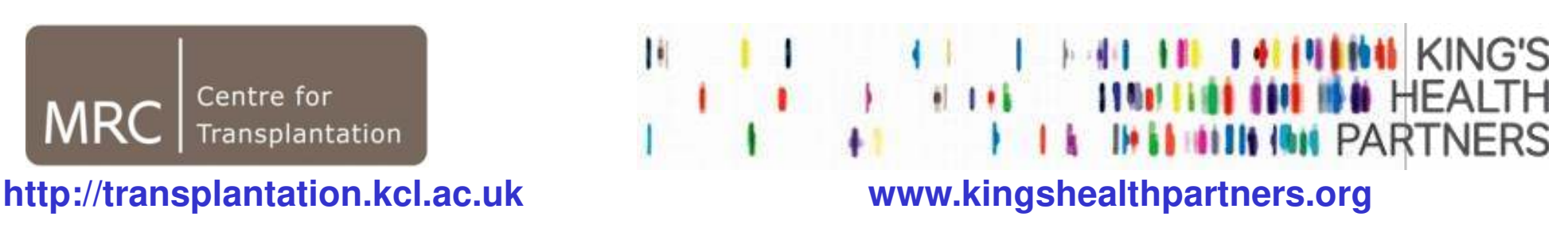

